\title{
Non-iterative Bit Loading Algorithm for OFDM in Independent and Correlated fading
}

\author{
John W. Manry* and Santosh Nagaraj**
}

\begin{abstract}
This paper will focus on improving the performance of orthogonal frequency division multiplexing (OFDM) in Rayleigh fading environments. The proposed technique will use a previously published method that has been shown to improve OFDM performance in independent fading, based on ordered sub-carrier selection. Then, a simple non-iterative method for finding the optimal bit-loading allocation was proposed. It was also based on ordered sub-carrier selection. We compared both of these algorithms to an optimal bit-loading solution to determine their effectiveness in a correlated fading environment. The correlated fading was simulated using the JTC channel models. Our intent was not to create an optimal solution, but to create a low complexity solution that can be used in a wireless environment in which the channel conditions change rapidly and that require a simple algorithm for fast bit loading.
\end{abstract}

Keywords-Adaptive Modulation, Orthogonal Frequency Division Multiplexing (OFDM), Fading

\section{INTRODUCTION}

Several of today's wireless standards have adopted Orthogonal Frequency Division Multiplexing (OFDM) as their multi carrier modulation technique [1,2]. This is due in part to the high resilience that OFDM has to Inter Symbol Interference (ISI), as well as for its ease of implementation. OFDM consists of several sub-carriers, each of which has an assigned symbol that undergoes an Inverse Fast Fourier Transform (IFFT) before being modulated to the assigned carrier frequency. This is often accompanied by the use of a cyclic prefix. The cyclic prefix is a copy of the end of the OFDM symbol, which is attached to the beginning of the symbol to protect it from ISI due to the effects of multi-path delay.

Multipath delay is caused by reflections of the original signal arriving at the receiver at different times [3]. While the cyclic prefix protects the OFDM symbol from the effect of ISI and multi-path delay, it does not mitigate the deep fades that can accompany multi-path delay [4]. Generally, OFDM symbols are equally loaded with the same symbol rate on each channel [2]. This means that the symbols on the faded channels will have a much higher bit error rate. To combat this, several adaptive techniques have been developed that alter the symbol rate on each channel according to the fading associated with the channel [5]. However, most of these techniques require complex algorithms that are too slow to keep up with the fast changing channels associated with wireless communications.

\footnotetext{
Manuscript received April 19, 2013; first revision July 21, 2013; accepted November 17, 2013. Corresponding author: Santosh Nagaraj (snagaraj@mail.sdsu.edu)

* Naval Air Systems Command, San Diego, CA 92019, U.S.A. (john.manry@navy.mil)

** Department of Electrical and Computer Engineering, San Diego State University, San Diego, CA 92182, U.S.A. (snagaraj@mail.sdsu.edu)
} 
The Ordered Sub-carrier Selection Algorithm (OSSA) has been proposed as a method of minimizing the effects from fading in independent Rayleigh fading environments [6]. This method uses a higher, uniform constellation size on only those channels with the highest gain. The constellation size is chosen in such a way that the data rate remains the same as the previous constellation size. This eliminates the need for complex calculations in order to determine the maximum constellation size for a given channel, as all the channels have the same rate. This method was shown to significantly reduce the bit error rate probability as compared to the conventional method of equal bit loading.

This paper will consider the performance of OFDM in correlated Rayleigh fading. The algorithms here will be based on bit loading techniques, while keeping the gain constant, based on the number of sub-carriers used. The total transmitted bit rate and power will remain constant, while the symbols per channel will be allocated in such a manner so as to minimize the total BER.

The first algorithm will minimize the BER of the system by determining the optimum combination of symbols to use. This will be done by loading the sub-carriers with the highest gain with the most bits per symbol and by loading the sub-carriers with the lowest gain with the fewest or none at all. This will ensure that the sub-carrier with the worst BER for each constellation group used is the same. The total bit rate of the system will remain unchanged. This algorithm will be accomplished using an iterative solution.

The second algorithm will be based on the same principle as the first algorithm, but it will be applied to the OSSA. Because OSSA uses a limited number of sub-carriers with a constant symbol rate on each channel, it will be possible to determine the optimum symbol allocation without the need for an iterative algorithm. By limiting the total number of different symbols to three, the BER of the two highest symbols can be compared to determine the optimal symbol allocation. The goal is to provide an algorithm that will perform well in both correlated and independent fading. As OSSA has already proven to be effective in independent fading, combining these techniques should accomplish that goal. This algorithm will also be applied to the conventional case in which all of the channels are used.

The algorithms proposed require the use of multiple symbols to increase the complexity required by the transmitter to modulate and assign multiple symbols as compared to OSSA. However, the performance improvement in correlated fading will make the overall system much more robust and the simplicity of the algorithm should keep the overall complexity down.

This paper will proceed as follows: the base system architecture, the reference scheme, and OSSA, will be described in Section II. Section III will describe the iterative algorithm in which the optimum BER will be found using adaptive bit loading. Section III will also cover the algorithm that will provide a non-iterative improvement to OSSA. Section IV will present the results. Finally this paper will conclude with the conclusion in Section V.

\section{SYSTEM DESCRIPTION}

\subsection{Base System}

The base system used is OFDM with $\mathrm{N}$ total sub-carriers. Each sub-carrier will be loaded with a symbol using Quadrature Amplitude Modulation (QAM) with a constellation size $\mathrm{m}_{\mathrm{i}}$, where 
$\mathrm{m}_{1}<\mathrm{m}_{2} \ldots<\mathrm{m}_{\mathrm{i}}$ and $\mathrm{m}$ is the total number of symbols possible in the M-QAM constellation. The constellation used is based on the number of bits per symbol, $\mathrm{k}_{\mathrm{i}}$, where $\mathrm{m}_{\mathrm{i}}=2^{\mathrm{ki}}$, and the values for $\mathrm{k}$ are even numbers, $(0,2,4,6 \ldots)$. The carriers will then be converted to the time domain with an $\mathrm{N}$ point inverse fast Fourier transform. The cyclic prefix will be created using $\mathrm{N}_{\mathrm{G}}$ samples from the end of the OFDM symbol and will be placed at the front of the OFDM symbol. This reduces the overall efficiency of the OFDM symbol by $\eta$, equation (1).

$$
\eta=\frac{N}{N+N_{G}}
$$

The signal is then transmitted over the wireless channel. The channel characteristics can be represented by Equation (2). $\mathrm{H}_{\mathrm{n}}$ represents the Rayleigh fading channel, where $\mathrm{n}$ corresponds to the $n^{\text {th }}$ sub-carrier. $X_{n}$ is the frequency representation of the transmitted symbol on the $n^{\text {th }}$ subcarrier of the OFDM symbol. The received signal is $Y_{n}$ and $n_{o}$ is the All White Gaussian Noise (AWGN). Using this model, the bit error probability of $\mathrm{P}_{\mathrm{b}}$, can then be calculated using Equation (3) and (4), [7]. Where $E_{b}$ is the total bit energy and $N_{o}$ is the variance of the AWGN, $\mathrm{n}_{0}$.

$$
\begin{gathered}
Y_{n}=H_{n} X_{n}+n_{o} \\
P_{b n} \sim \frac{2\left(1-L^{-1}\right)}{\log _{2} L} Q\left[\sqrt{\left(\frac{3 \log _{2} L}{L^{2}-1}\right) \frac{2\left|H_{n}\right|^{2} \eta E_{b}}{N_{o}}}\right] \\
L=\sqrt{m_{i}}
\end{gathered}
$$

\subsection{Ordered Subcarrier Selection Algorithm}

The authors of [6] determined that in an independent Rayleigh fading environment there were an optimal number of channels, $\mathrm{K}_{\mathrm{opt}}$, to use. This was determined using Equation (3) and the Rayleigh distribution function to create Equation (5). In Equation 5, $\mathrm{m}_{\mathrm{ref}}$ refers to the constellation, $\mathrm{m}_{\mathrm{i}}$, which is used in conventional OFDM, in which all the channels are equally loaded with one symbol size.

$$
K_{o p t}=\frac{N \log _{2}\left(m_{r e f}\right)}{\log _{2}\left(4 m_{r e f}\right)}
$$

The new constellation of $\mathrm{m}$ symbols that is required to keep the transmitted bit rate the same as the base system on $\mathrm{K}_{\mathrm{opt}}$ channels can be determined from Equation (6).

$$
m=m_{r e f} 2^{N / K o p t}
$$




\section{Proposed Algorithms}

\subsection{Iterative Algorithm}

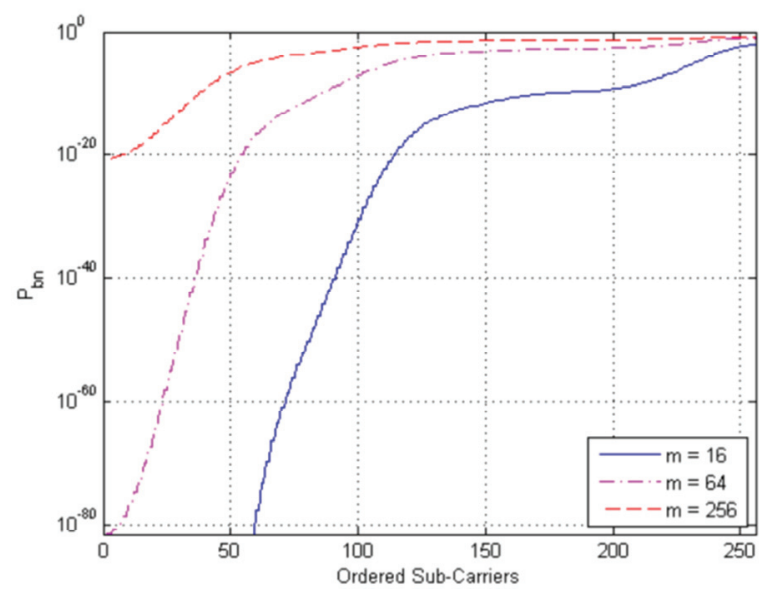

Fig. 1. $P_{b n}$ for several constellations ordered according to the channel gain per subcarrier from Equation (7). The total number of sub-carriers used was 256 with $E_{b} / N_{\circ}$ of $20 \mathrm{~dB}$

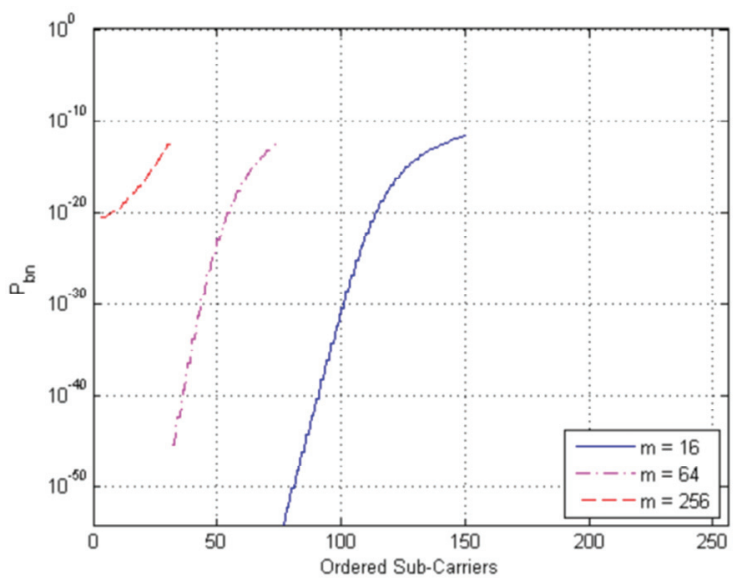

Fig. 2. $P_{b n}$ for the selected number of constellations that will provide the lowest possible $B E R$, as found from the iterative algorithm. The total number of sub-carriers used was 256 with $\mathrm{E}_{\mathrm{b}} / \mathrm{N}_{\mathrm{o}}$ of $20 \mathrm{~dB}$ 


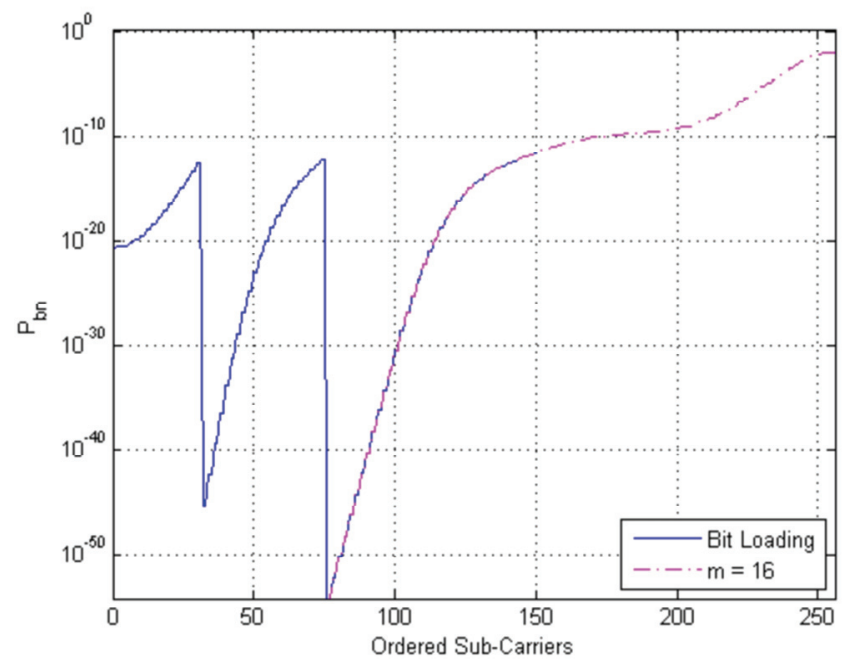

Fig. 3. $\mathrm{P}_{\mathrm{bn}}$ for the bit loaded signal versus a baseline transmission scheme as a function of the ordered subcarrier index. With the proposed bit-loading, fewer subcarriers are used, exhibiting a lower bit error probability. The total number of sub-carriers used was 256 with $E_{b} / N_{o}$ of $20 \mathrm{~dB}$

This algorithm will find the optimum number of symbols to transmit per sub-carrier so that the data transmission rate does not change. The total power transmitted remains the same and the BER is reduced to the lowest possible value. This assumes that the transmitter has knowledge of the channel. The channel gain, $H_{n}$, is then ordered using Equation (7).

$$
H_{1}>H_{2} \ldots>H_{N} \quad \text { for } n=1,2, \ldots, N
$$

Using Equation (3), the $\mathrm{P}_{\mathrm{bn}}$ for $\mathrm{m}_{\mathrm{i}}, \mathrm{m}_{\mathrm{i}+1}$ and $\mathrm{m}_{\mathrm{i}+2}$ using the sub-carrier channel gains, as ordered in Equation (7), is found. This is shown in Figure 1. $\mathrm{P}_{\mathrm{bN}}\left(\mathrm{m}_{\mathrm{i}}\right)$, the probability of a bit error occuring on the $256^{\text {th }}$ ordered sub-carrier is then compared to $\mathrm{P}_{b n}\left(\mathrm{~m}_{\mathrm{i}+1}\right)$ and $\mathrm{P}_{\mathrm{bn}}\left(\mathrm{m}_{\mathrm{i}+2}\right)$. The total number of channels assigned to $\mathrm{m}_{\mathrm{i}+2}, \mathrm{~N}_{\mathrm{mi}+2}$ is equal to the number of channels for which $\mathrm{P}_{\mathrm{bn}}\left(\mathrm{m}_{\mathrm{i}+2}\right)<\mathrm{P}_{\mathrm{bN}}\left(\mathrm{m}_{\mathrm{i}}\right)$ for all $\mathrm{n}$, (see Equation (8)).

$$
N_{m_{i+2}}=\sum_{j=1}^{N}\left(\mathrm{P}_{\mathrm{b}_{\mathrm{j}}}\left(\mathrm{m}_{\mathrm{i}+2}\right)<\mathrm{P}_{\mathrm{b}_{\mathrm{N}}}\left(\mathrm{m}_{\mathrm{i}}\right)\right)
$$

Here, the notation $(a<b)$ represents the indicator function. It returns ' 1 ' if the statement ' $a<b$ ' is true and ' 0 ' otherwise. Similarly, the total number of channels assigned to $m_{i+1}, N_{m i+1}$ is found using Equation (9).

$$
N_{m_{i+1}}=\sum_{j=1}^{N}\left(\mathrm{P}_{\mathrm{b}_{\mathrm{j}}}\left(\mathrm{m}_{\mathrm{i}+1}\right)<\mathrm{P}_{\mathrm{b}_{\mathrm{N}}}\left(\mathrm{m}_{\mathrm{i}}\right)\right)
$$

If $\mathrm{N}_{\mathrm{mi}+2}$ is greater than $\mathrm{N}_{\mathrm{mi+1}+1}$, then the number of sub-carriers assigned is found using Equation (10), otherwise no subcarriers are assigned. 


$$
N_{m i+2, \text { new }}=N_{m i+2}-N_{m i+1}
$$

The remaining channels are assigned to $\mathrm{m}_{\mathrm{i}}$. So,

$$
N_{i}=N-N_{m i+1}-N_{m i+2}
$$

The total number of bits to be transmitted, $b_{\text {tnew }}$, is then determined using Equation (12).

$$
b_{t, \text { new }}=k_{i+2} N_{m i+2}+k_{i+1} N_{m i+1}+k_{i} N_{m i}
$$

If this number is greater than the original number of bits, $b_{t}$, then the process is repeated using a new value for the total number of sub-carriers found using Equation (14).

$$
\begin{gathered}
b_{t}=k_{i} N \\
N_{\text {new }}=N-1
\end{gathered}
$$

This process will continue until the bit rate to be transmitted is equal to the original bit rate. At each step of the iterative algorithm, the $\mathrm{P}_{\mathrm{b}}(\mathrm{m})$ for the sub-carrier with the worst gain, $\mathrm{H}_{\mathrm{n}}$, for each constellation is approximately the same (see Figure 2).

This process iteratively replaces sub-carriers with a high BER with sub-carriers with lower $\mathrm{BER}$, driving the overall BER to its lowest possible value given the constraints. Figure 3 shows that the total number of used sub-carriers has been reduced from 256 to 150 . Also, the lowest $\mathrm{P}_{\mathrm{b}}$ is now approximately $10^{\wedge}-13$ versus the previous low of $10^{\wedge}-3$. This is a significant reduction in transmitted BER. However, this comes with a cost of 100 iterations. This algorithm will be used to evaluate the performance of the next algorithm and OSSA.

\subsection{Non-Iterative Algorithm}

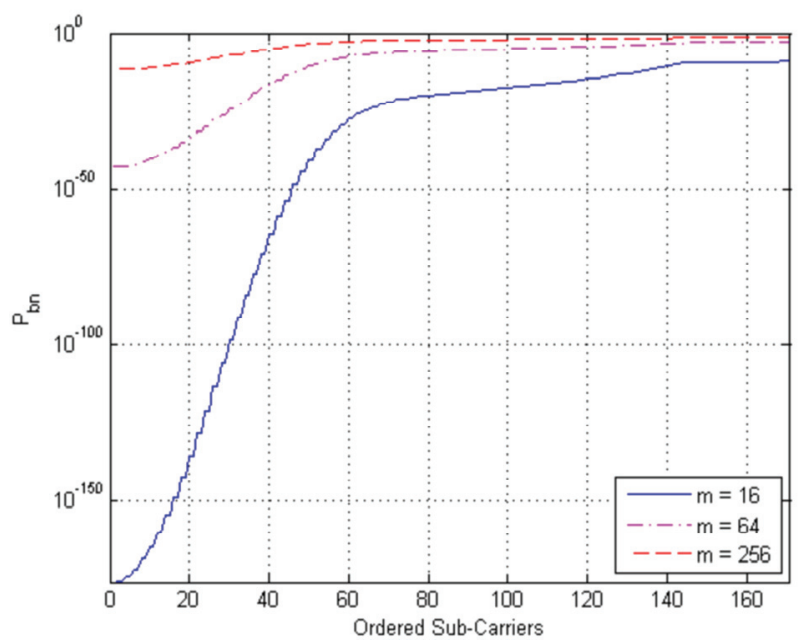

Fig. 4. The $P_{b n}$ for several constellations ordered according to the channel gain per sub-carrier from Equation (15). The total number of sub-carriers used was 256 . However, only $k_{\text {opt }}$ subcarriers are shown, with $E_{b} / N_{o}$ of $20 d B$ 


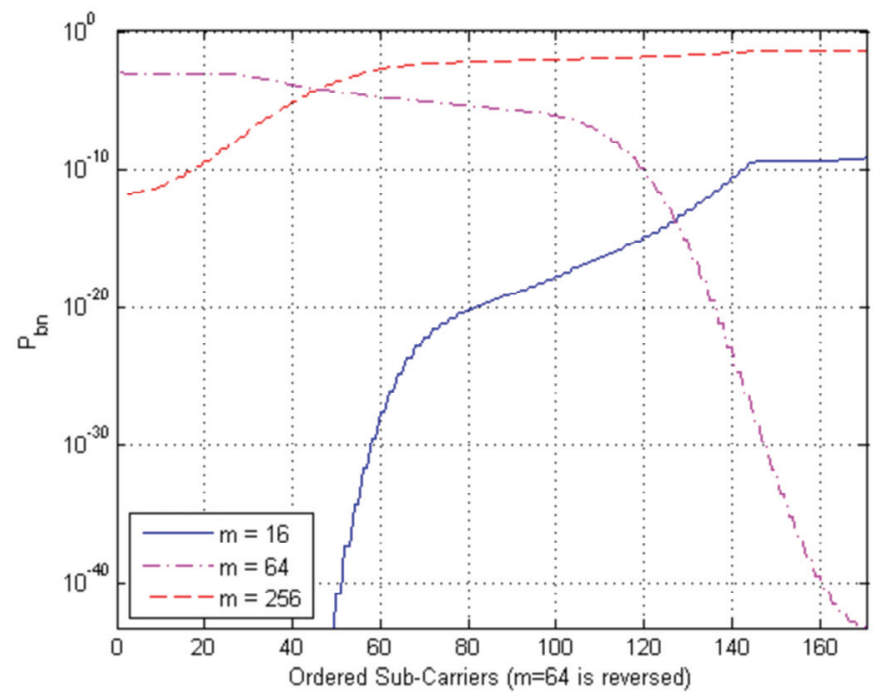

Fig. 5. The $P_{b n}$ for several constellations with $m=64$ reversed. It can be seen that the $P_{b n}$ for $m=64$ is higher than that for $m=256$ over 46 channels. The total number of sub-carriers used was 256. However, only $k_{\text {opt }}$ sub-carriers are shown, with $E_{b} / N_{\circ}$ of $20 d B$

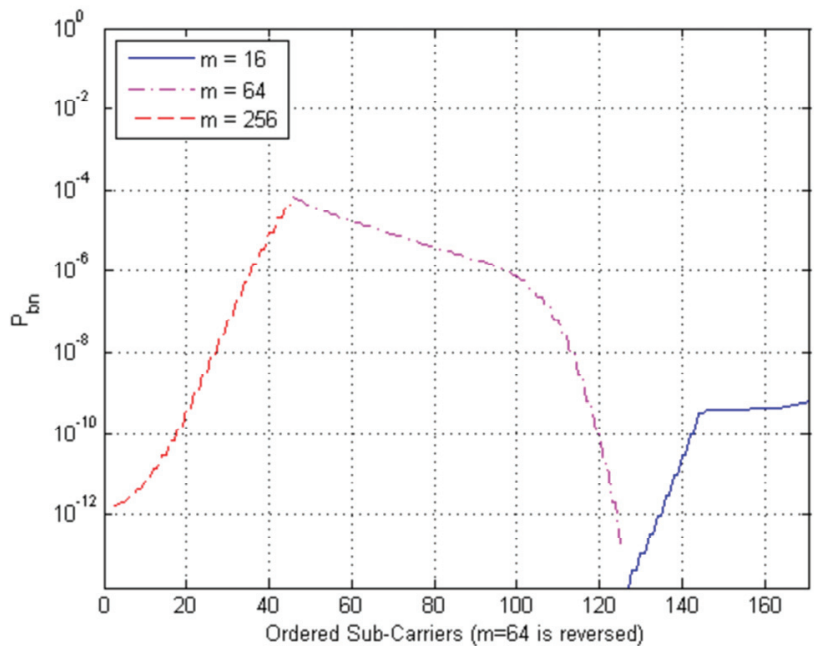

Fig. 6. The $P_{b n}$ for each sub-carrier loaded with the proper constellation as determined by the noniterative algorithm 


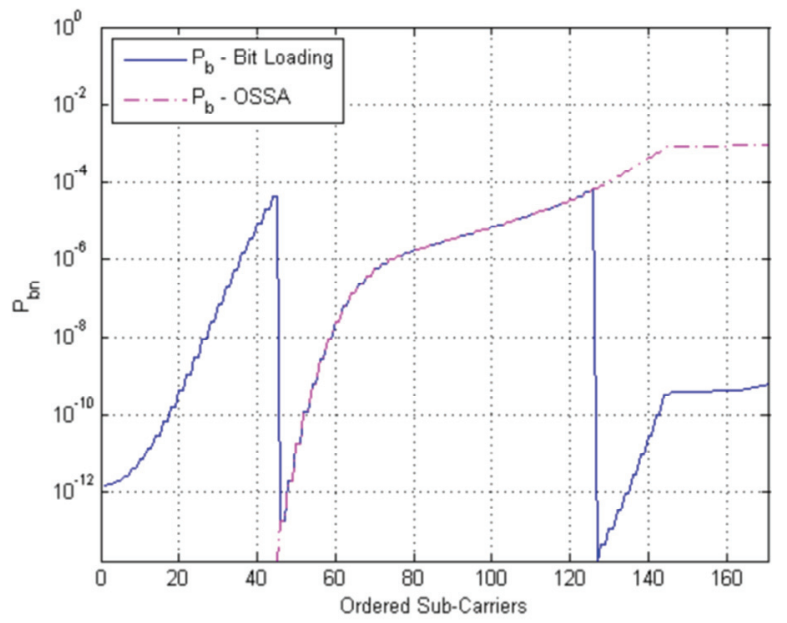

Fig. 7. The $P_{b n}$ for the bit loaded signal versus the original signal using OSSA

OSSA uses the same constellation size over all $\mathrm{K}_{\mathrm{opt}}$ sub-carriers. If the constellation size is restricted to $\mathrm{m}_{\mathrm{i}-1}, \mathrm{~m}_{\mathrm{i}}$, and $\mathrm{m}_{\mathrm{i}+1}$, where $\mathrm{m}_{\mathrm{i}}$ is the constellation size used by OSSA, and the number of channels used is always $\mathrm{K}_{\mathrm{opt}}$, the optimal distribution for these symbols can be found without iteration. The algorithm will work by evaluating the sum of the sub-carriers with the highest $\mathrm{P}_{\mathrm{b}}\left(\mathrm{m}_{\mathrm{i}}\right)$ and the lowest $\mathrm{P}_{\mathrm{b}}\left(\mathrm{m}_{\mathrm{i}}\right)$. This will be compared to the sum of $\mathrm{P}_{\mathrm{b}}\left(\mathrm{m}_{\mathrm{i}-1}\right)$ and $\mathrm{P}_{\mathrm{b}}\left(\mathrm{m}_{\mathrm{i}+1}\right)$ where the higher constellation rate is loaded onto the sub-carrier with the highest gain, and the lower constellation rate is loaded onto the sub-carrier with the lowest channel gain. If the combined $\mathrm{P}_{\mathrm{b}}\left(\mathrm{m}_{\mathrm{i}}\right)$ is greater than the combined $\mathrm{P}_{\mathrm{b}}\left(\mathrm{m}_{\mathrm{i}-1}\right)$ and $\mathrm{P}_{\mathrm{b}}\left(\mathrm{m}_{\mathrm{i}+1}\right)$, the current symbols will be replaced by the symbols providing the lowest $\mathrm{P}_{\mathrm{b}}$. If that is not the case, the symbols will remain the same and the algorithm will end.

The total number of channels, $\mathrm{N}_{\mathrm{m}}$ to use for each symbol can be found by first ordering the channel gains using Equation (15). Equation (3) is used to find the $\mathrm{P}_{\mathrm{b}}(\mathrm{m})$ for each constellation using the ordered sub-carrier channel gains, as shown in Figure 4. Next, the number of higher constellation symbols, $\mathrm{N}_{\mathrm{mi}+1}$, is determined using Equation (16).

$$
\begin{gathered}
H_{1} \leq H_{2} \leq \cdots H_{n} ; \text { For } n=1,2, \ldots, K_{o p t} \\
N_{m_{i+1}}=\sum_{j=1}^{\frac{K_{o p t}}{2}}\left(P_{b_{K_{o p t}-j}}\left(m_{i}\right)+P_{b_{j}}\left(m_{i}\right)>P_{b_{K_{o p t}-j}}\left(m_{i+1}\right)+P_{b_{j}}\left(m_{i-1}\right)\right)
\end{gathered}
$$

Figure 5 shows the $\mathrm{P}_{\mathrm{bn}}$ for constellation sizes 16, 64, and 256. Unlike Figure 4, the plot for constellation size 64 has been reversed to show the difference between the $\mathrm{P}_{\mathrm{bn}}$ for constellation size 64 with the lowest $H_{n}$ and constellation size 256 with the highest $H_{n}$. Where the two plots intersect, in this case at $n=46$, is the number of carriers, $N_{m i+1}$, found from Equation (16) to provide the lowest possible BER. The number of symbols for $\mathrm{m}_{\mathrm{i}-1}$ and $\mathrm{m}_{\mathrm{i}}$ is determined using Equations (17) and (18).

$$
N_{m_{i-1}}=N_{m_{i+1}}
$$




$$
N_{m_{i}}=K_{\text {opt }}-2 N_{m_{i+1}} ; \text { If }\left(K_{\text {opt }}-2 N_{m_{i+1}}\right)>0
$$

Otherwise:

$$
N_{m_{i}}=0
$$

Figure 6 shows the $\mathrm{P}_{b n}$ for each sub-carrier that will be transmitted as a result of this algorithm. Because the $\mathrm{P}_{\mathrm{b}}$ of the symbols being replaced is higher than replacement symbols, the total BER will always be lower. In Figure 7, this can be seen by noting that the lowest $\mathrm{P}_{\mathrm{bn}}$ of OSSA is $10^{\wedge}-3$, while the lowest $\mathrm{P}_{\mathrm{bn}}$ of the bit loaded signal is $10^{\wedge}-4$. This is a huge improvement over OSSA. The algorithm ensures that the lowest BER is obtained without the need for multiple iterations. This also ensures that the total bit rate of the OFDM symbol remains constant, as the number of symbols being removed is equal to the number of symbols being added.

This algorithm can be further simplified by making the following observations: first, the probability of bit error for constellation $\mathrm{m}_{\mathrm{i}}$, will always be higher on channels with lower gain than on channels with higher gain, (see Figure 4, $\mathrm{m}=64$ ). This can be expressed by Equation (20) for small $j$. Equation (20) can then be used to make the approximation shown in Equation (21).

$$
\begin{aligned}
P_{b_{K_{o p t}-j}}\left(m_{i}\right) & \gg P_{b_{j}}\left(m_{i}\right) \\
P_{b_{K_{o p t}-j}}\left(m_{i}\right)+P_{b_{j}}\left(m_{i}\right) & \approx P_{b_{K_{o p t}-j}}\left(m_{i}\right)
\end{aligned}
$$

Second, as $j$ increases in Equation (16), the assumptions in Equations (22) and (23) can be made.

$$
\begin{gathered}
P_{b_{K_{o p t^{-j}}}}\left(m_{i+1}\right) \geq P_{b_{K_{o p t^{-j-1}}}\left(m_{i+1}\right)} \\
P_{b_{j}}\left(m_{i-1}\right) \leq P_{b_{j-1}}\left(m_{i-1}\right)
\end{gathered}
$$

From Equations (22) and (23), the approximation in Equation (24) can be made.

$$
P_{b_{K_{o p t}-j}}\left(m_{i+1}\right)+P_{b_{j}}\left(m_{i-1}\right) \approx P_{b_{K_{o p t}-j}}\left(m_{i+1}\right)
$$

Substituting the results of Equations (21) and (24) into Equation (16), Equation (16) can be rewritten as Equation (25). This form reduces the complexity of the original algorithm by eliminating the need to add up the total probability of a bit error occurring where all of the symbols are replaced.

$$
N_{m_{i+1}}=\sum_{j=1}^{\frac{K_{o p t}}{2}}\left(P_{b_{j}}\left(m_{i}\right)>P_{b_{K_{o p t}-j}}\left(m_{i+1}\right)\right)
$$

This form of the algorithm no longer requires the extra addition of probabilities and shows that it is only necessary to compare the worst values of $\mathrm{P}_{b}\left(\mathrm{~m}_{\mathrm{i}}\right)$ with the best values of $\mathrm{P}_{\mathrm{b}}\left(\mathrm{m}_{\mathrm{i}+1}\right)$ to obtain the optimal solution. In other words, for every sub-carrier with symbol $\mathrm{m}_{\mathrm{i}}$ with a lower $\mathrm{P}_{\mathrm{b}}$ than a sub-carrier with symbol $\mathrm{m}_{\mathrm{i}+1}$, the sub-carrier with the highest gain is loaded with $\mathrm{m}_{\mathrm{i}+1}$ and 
the sub-carrier with the lowest channel gain is loaded with $\mathrm{m}_{\mathrm{i}-1}$. Combing this algorithm with OSSA will create a more robust system while keeping the overall complexity low.

\section{Simulation Results}

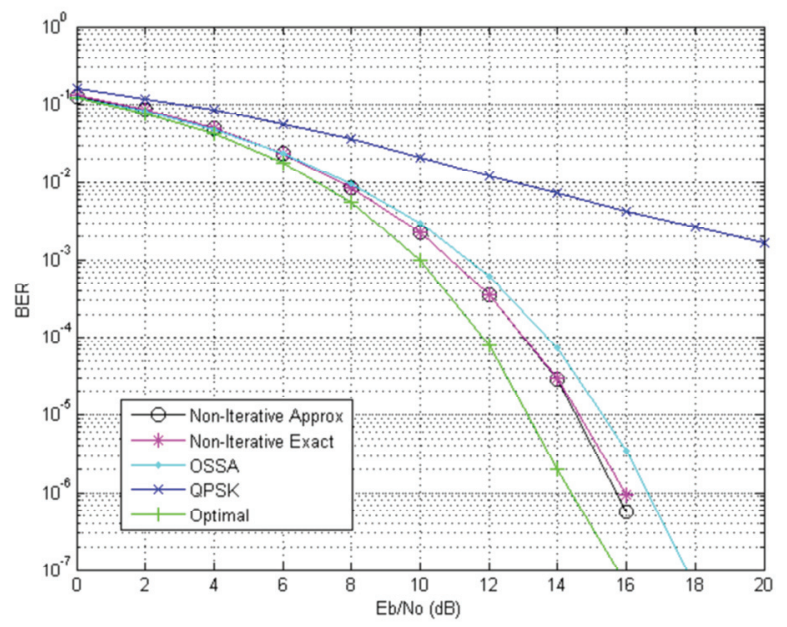

Fig. 8. Performance of several algorithms versus QPSK in the JTC simulation model for an indoor office environment

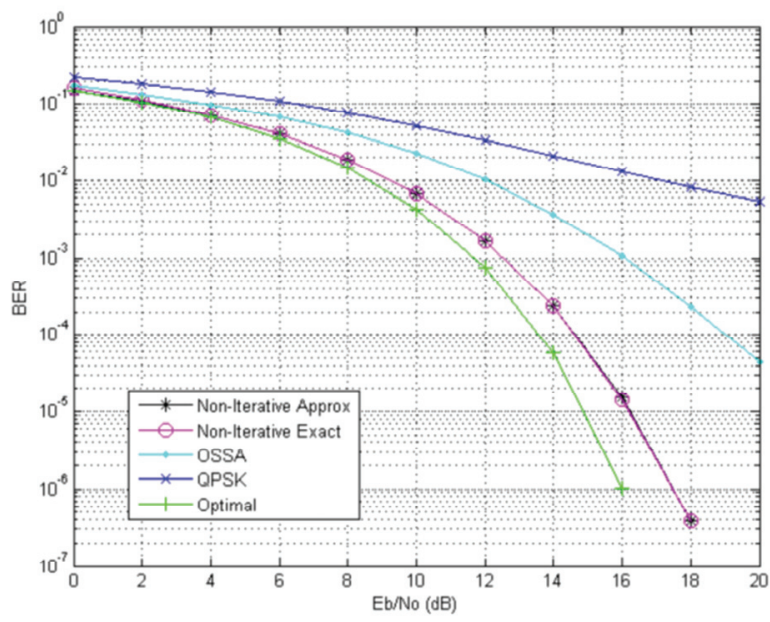

Fig. 9. Performance of several algorithms versus QPSK in the JTC simulation model for an outdoor urban high-rise profile 


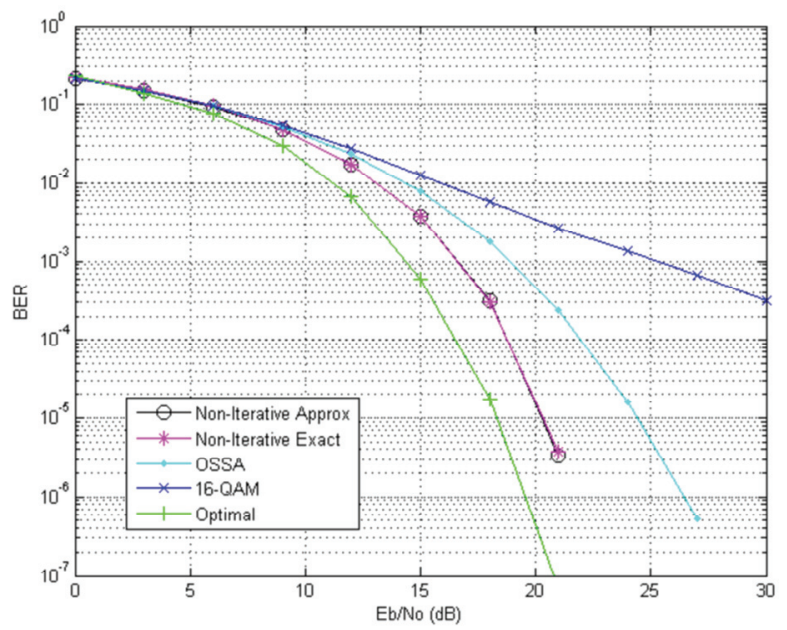

Fig. 10. Performance of several algorithms versus 16-QAM in the JTC simulation model for an indoor office environment

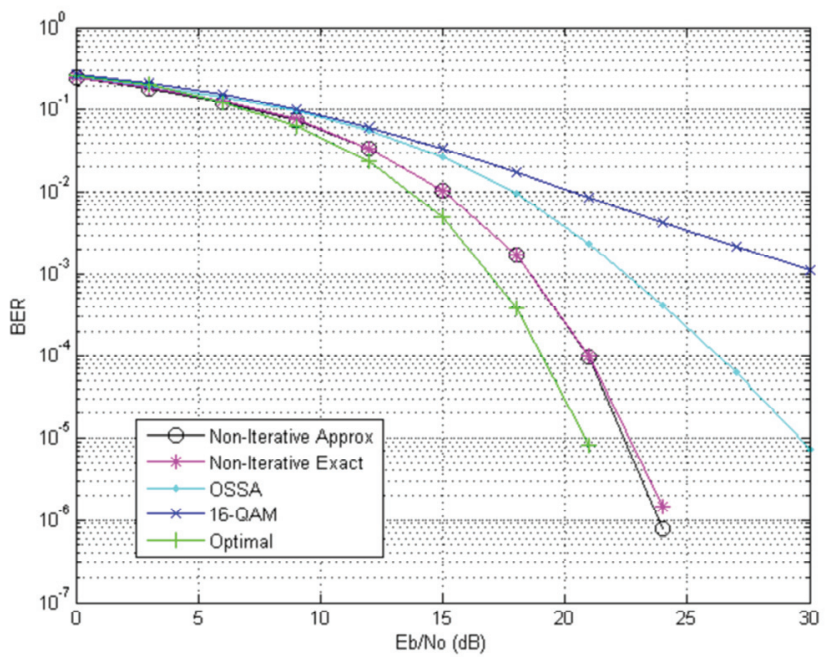

Fig. 11. Performance of several algorithms versus 16-QAM in the JTC simulation model for an outdoor urban high-rise profile

The simulations will use OFDM symbols with 256 sub-carriers. Two JTC realistic channel models will be used $[8,9]$. These will be the Indoor Office profile and the outdoor urban highrise profile. The JTC profiles will simulate the correlated multipath fading channel, which will be used to evaluate the performance of the algorithms. The iterative algorithm will be used as an optimal solution to compare the performance of OFDM, OSSA, and the non-iterative OSSA improvement algorithm with both the approximate solution and the exact solution. The last two will be labeled as the Non-Iterative Approx and the Non-Iterative Exact.

The first simulation uses the JTC indoor profile. The reference scheme is for a constellation size of 4, or QPSK. In this scheme OSSA will use 128 channels loaded with a constellation size of 16 . The number of channels used by the optimal scheme varies, and the number of channels 
used by the non-iterative solutions is the same as OSSA. From Figure 8, it can be seen that all schemes exhibit a huge performance increase versus QPSK. Both the optimal scheme and the non-iterative schemes outperform OSSA. However, the non-iterative scheme has only a $1 \mathrm{~dB}$ performance gain, while the optimal scheme shows a $2 \mathrm{~dB}$ improvement over OSSA. The difference between the optimal scheme and the non-iterative schemes is slightly over $1 \mathrm{~dB}$.

Figure 9 shows the results for the reference scheme of QPSK in a JTC outdoor urban high-rise environment. This exhibits more correlated fading than that in the JTC indoor office environment. The performance gain of OSSA over QPSK somewhat decreases. However, OSSA still exhibits great improvement over QPSK. Both non-iterative algorithms show a $5 \mathrm{~dB}$ improvement over OSSA at a BER of $10^{\wedge}-4$. This is a huge gain at a relatively low computational complexity. The optimal solution is the best, and shows a $1 \mathrm{~dB}$ improvement over the non-iterative solution. It should also be noted that in both cases, the non-iterative approximation performs identically to the non-iterative exact solution. This confirms that the approximations made in Equation (25) are indeed valid.

The results for the reference scheme of 16-QAM in the JTC indoor environment are shown in Figure 10. All of the schemes outperform the reference scheme. The non-iterative schemes show a performance of more than a $4 \mathrm{~dB}$ increase over OSSA at a BER of $10^{\wedge}-5$. The optimal scheme outperforms the non-iterative scheme by $2 \mathrm{~dB}$. This shows a much greater performance increase versus OSSA than in the indoor profile with the reference scheme of QPSK. This clearly demonstrates that the performance of the non-iterative algorithm improves as the bit rate increases, as compared to OSSA.

Figure 11 shows the performance for the algorithms with the reference scheme of 16-QAM in the JTC outdoor profile. The performance of both the non-iterative algorithms and the optimal algorithm remain relatively constant for that of the indoor scheme. While the performance of the reference system and OSSA suffer much more degradation. The improvement of the noniterative schemes over OSSA is $8 \mathrm{~dB}$. The performance of the optimal scheme over the noniterative scheme remains constant at $2 \mathrm{~dB}$.

\section{Conclusions}

OSSA was shown to be an effective solution to independent Rayleigh fading in [6]. It was also shown to provide an improvement in correlated fading over the baseline system. This paper has shown that by including a very simple bit loading scheme with OSSA, a much more robust solution to both correlated fading and independent fading could be created. While the iterative and non-iterative schemes discussed in this paper will add a complexity increase in the form of requiring multiple modulations on the same OFDM symbol, the overall complexity of the scheme is very simple. The improvement of OSSA alone was significant in all but the lowest fading conditions. The performance loss over the optimal bit loading scheme was constant at $1 \mathrm{~dB}$ for a low bit rate scheme and $2 \mathrm{~dB}$ for a high bit rate scheme. This was very small compared to the performance gain by the non-iterative algorithm over just OSSA alone. The non-iterative scheme would also provide an ideal solution for an outdoor channel that requires an algorithm that can be quickly updated to perform in a fast changing wireless channel. An exact analysis of the complexity of this algorithm is an avenue for future work. 


\section{REFERENCES}

[1] IEEE Standard for Local and Metropolitan Area Networks, IEEE Standard 802.16e, 2005.

[2] IEEE Standard for Information Technology-Telecommunications and Information Exchange Between Systems-Local and Metropolitan Area Networks, IEEE Standard 802.11g, 2003.

[3] C. L. Holloway, M. G. Cotton, and P. McKenna, "A simplified model for predicting the power delay profile characteristics of an indoor radio propagation channel," Institute for Telecommunication Sciences, Boulder, CO, NTIA Technical Report TR-98-353, August 1998.

[4] T. R. Rao and A. Giulietti, "A performance study on the 802.11g WLAN OFDM system," in The International Conference on Computer as a Tool, Belgrade, Serbia and Montenegro, 2005, pp. 128130.

[5] J. A. C. Bingham, "Multicarrier modulation for data transmission: an idea whose time has come," IEEE Communications Magazine, vol. 28, no. 5, pp. 5-14, 1990.

[6] D. Dardari, "Ordered subcarrier selection algorithm for OFDM-based high-speed WLANs," IEEE Transactions on Wireless Communications, vol. 3, no. 5, pp. 1452-1458, 2004.

[7] B. Sklar, Digital Communications: Fundamentals and Applications, 2nd ed. Upper Saddle River, NJ: Prentice-Hall PTR, 2001.

[8] Deployment/Testing Ad Hoc Group, Joint Technical Committee (AIR) Standards Contribution, September 1994. Joint Technical Committee (Air) Standards Contribution, "Technical report on RF channel characterization and system deployment modeling," JTC (Air) Standards Contribution, Technical Report JTC(AIR)/94.09.23-065R6, September 1994.

[9] D. S. Kim, S. H. Kim, C. S. Kim, and M. M. Lee, "Performance analysis of OFDM system based on IEEE 802.11a," in Proceedings of the International Technical Conference on Circuits, Systems, Computers and Communications, Phuket, Thailand, 2002.

\section{John W. Manry}

He received the MS degree in Electrical and Computer Engineering from San Diego State University in 2008. His MS research was on designing efficient adaptive modulation techniques for OFDM based radios. He currently works for the Naval Air Systems Command in San Diego, California, United States of America.

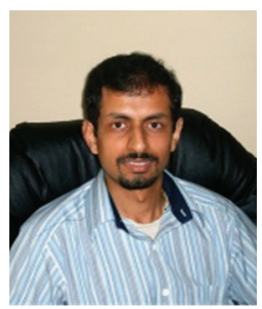

\section{Santosh Nagaraj}

He received the Ph.D. degree in Electrical Engineering from Purdue University in 2005. Since then, he has been a faculty in the Department of Electrical and Computer Engineering at San Diego State University in San Diego, California. His research interests include wireless modems, error correcting codes and information theory. 